\title{
Local dimension and finite time prediction in spatiotemporal chaotic systems
}

\author{
Gerson Francisco* and Paulsamy Muruganandam ${ }^{\dagger}$ \\ Instituto de Física Teórica, Universidade Estadual Paulista, 01405-900 São Paulo, SP, Brazil
}

(Received 06 December 2002; published 12 June 2003)

\begin{abstract}
We show how a recently introduced statistic [Patil et al., Phys. Rev. Lett. 81, 5878 (2001)] provides a direct relationship between dimension and predictability in spatiotemporal chaotic systems. Regions of low dimension are identified as having high predictability and vice versa. This conclusion is reached by using methods from dynamical systems theory and Bayesian modeling. In this work we emphasize on the consequences for short time forecasting and examine the relevance for factor analysis. Although we concentrate on coupled map lattices and coupled nonlinear oscillators for convenience, any other spatially distributed system could be used instead, such as turbulent fluid flows.
\end{abstract}

DOI: 10.1103/PhysRevE.67.066204

PACS number(s): 05.45.Jn, 05.45.Ra, 05.45.Tp

\section{INTRODUCTION}

Systems formed by aggregates of parts that interact in a nonlinear way are prototypes of complex behavior in physics, biology, and economics. An often successful approach in the analysis of such systems is to use tools from dynamical systems theory applied locally at points of spatially distributed configurations. In many cases, local nonlinearities lead to unpredictable chaotic evolution where short time forecasts are still feasible. An instance of this situation is atmospheric research where, for obvious reasons, prediction is often the most important goal to be achieved. In general, for large spatially distributed systems, it is highly desirable to have a simple diagnostic tool to identify regions of predictable behavior. The main aim of this paper is to show that certain spatial regions do indeed yield better forecasts than other locations. To elaborate on this theme, we use the concept of bred vector (BV) dimension, introduced in Ref. [1] in the context of the earth's atmosphere, as a tool to identify regions of high dimensionality. Our objective is then to show that this dimensional estimate is directly related to our ability to make short time forecasts. A recently introduced Bayesian approach, the cluster weighted modeling (CWM) [2], is one of the methods on which we base our conclusions. We start from a simple prediction algorithm [3] where the main idea of the paper is readily recognized. The more sophisticated CWM approach in conjunction with the simpler prediction algorithm will provide a clear link between dimension and our ability to forecast the behavior of deterministic systems. In particular, Bayesian approaches also permit the calculation of the variance (confidence intervals) of forecasts. In Ref. [4], we find a similar approach where a measure of predictability is based on the variance under the evolution of suitably defined functions in embedding space. Such an approach requires long evolution times, which cannot be afforded in the present context, and, as will be seen herein, this problem is circumvented by the use of the CWM.

\footnotetext{
*Electronic address: gerson@ift.unesp.br

${ }^{\dagger}$ Present address: Center for Nonlinear Dynamics, Department of Physics, Bharathidasan University, Tiruchirapalli 620024, Tamil Nadu, India.
}

The concept of correlation dimension $[5,6]$ has some bearing on this subject since high-dimensional systems cannot be easily distinguished from systems with dominant stochastic component, where predictability is known to be low. Here, by prediction we mean the existence of a deterministic map, which can be successfully used to make short time forecasts, in the least square sense or using some more general likelihood functions.

The paper is organized as follows. In Sec. II, we present the basic aspects and definitions of the BV dimension, factor analysis, and the necessary tools to relate dimension with predictability. In Sec. III, the spatiotemporal systems given by coupled logistic maps and coupled Lorenz systems are discussed, and the results of the simulations are presented. Our concluding remarks are outlined in Sec. IV.

\section{LOCAL DIMENSION AND PREDICTABILITY}

The notion of dimension used herein is based on the concept of bred vectors. They are constructed in a similar way as Lyapunov vectors, but in practical applications they differ in two aspects. First, for bred vectors there is no global orthonormalization, and second, they are finite amplitude, finite time vectors. Such properties facilitate the calculation of bred vectors and yield an efficient identification of regions where short time forecasts are feasible.

Consider a two-dimensional (2D) spatially distributed system whose state at a given time $t_{1}$ is defined over a collection of points $(i, j)$. Here we take the $M-1$ nearest neighbors for each point $(i, j)$ in a square lattice with $M$ $=25$, as illustrated in Fig. 1. Logistic maps are one variable dynamical systems, and in order to specify the corresponding state at a point including its neighbors, we need an $M$-dimensional state vector. Considering the $x-y$ coordinates of the Lorenz system specified at a suitable constant $z$ hyperplane, the state vector in this case requires $2 M$ components. In general, the state vector, either $M$ dimensional or $2 M$ dimensional, will be called bred vector. Now, generate $k$ distinct perturbations of the state starting at $t_{0}<t_{1}$ obtaining $k$ local bred vectors. The $k \times k$ covariance matrix of the system is just $\boldsymbol{C}=\boldsymbol{B}^{T} \boldsymbol{B}$, where $\boldsymbol{B}$ is the $M \times k$ (or $2 M \times k$ for the Lorenz system) matrix of local bred vectors each normalized to unity. In this paper, we will fix $k=5$. 


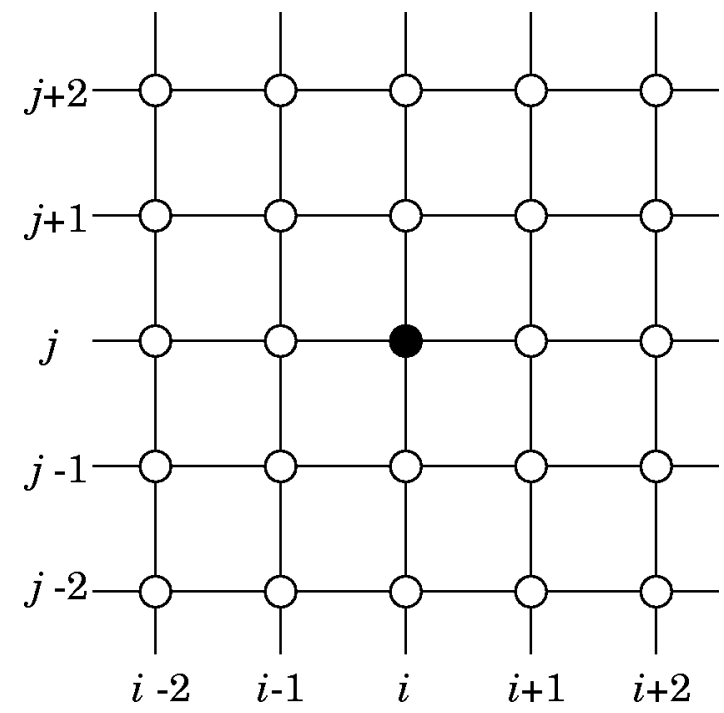

FIG. 1. Schematic diagram showing the choice of nearest neighbors at site $(i, j)$ for local dimension. The bred vectors are the dynamical variables associated with these sites.

We order the eigenvalues of the covariance matrix as $\lambda_{1}$ $\geqslant \lambda_{2} \geqslant \cdots \geqslant \lambda_{k}$ and define the singular values of $\boldsymbol{B}$ as $\sigma_{i}$ $=\sqrt{\lambda_{i}}$. Here the connection with factor analysis is clear [7], since the eigenvalues of the covariance matrix give an idea of the local linear independence of the $k$ local bred vectors. An effective dimension of the space of bred vectors can be obtained by fixing a threshold value corresponding to the highest $l$ eigenvalues, as is done in principal components analysis. Thus, an approximation of the data, supposing zero average for simplicity, is contained in the product $\mathcal{F} \boldsymbol{L}$, where $\mathcal{F}=\boldsymbol{B} \boldsymbol{L}^{T}$ is called the factor and $\boldsymbol{L}$ is the loading matrix of dimension $l \times k$. The rows of the loading matrix are the components of the eigenvectors corresponding to the dominant eigenvalues. Clearly, there is an arbitrariness in the stipulation of $l$, and this ambiguity is absent when using the concept of BV dimension.

The eigenvalues $\lambda_{i}$ represent the amount of variance in the set of the $k$ unit bred vectors. In order to estimate unambiguously the value of the threshold, one defines the following statistic [1]:

$$
\psi_{i, j}\left(\sigma_{1}, \sigma_{2}, \ldots, \sigma_{k}\right)=\frac{\left(\sum_{l=1}^{k} \sigma_{l}\right)^{2}}{\sum_{l=1}^{k} \sigma_{l}^{2}} .
$$

As each of the $k$ bred vectors is normalized to unity, $\psi$ assumes values in the interval $(0, k)$. Examples of the values of this statistic for several distributions of bred vectors can be found in Ref. [1]. A property of the statistic just defined is its robustness under noise or numerical errors. It can be used to determine the dominant eigenvalues $l$; just take it to be the smallest integer bigger than $\psi$. In this sense, an approximation to the bred vectors is obtained as the product of the corresponding factor and the loading matrix.
We describe now the criteria and tools that will be implemented in the following section to spatially distributed systems in order to obtain a connection between dimension and predictability. We start with a simple prediction algorithm that is very useful in the analysis of nonlinear time series [3]. To describe this criterion, consider a scalar time series obtained from a component of the dynamical variables, say, the $x$ component, defined at a given site in the 2D lattice. Using delay coordinates, one considers neighbors, within a distance smaller than $\delta$, to a given point in the embedding space. For the present analysis, the choice of embedding dimension three is adequate. By propagating the corresponding neighbors of a given vector in embedding space and averaging them, we obtain a forecast of the vector. The more sophisticated CWM [2] is a Bayesian approach where the embedded time series is used to build local Gaussian models. One of the advantages of this method is that it goes beyond point prediction since it includes errorbars (confidence intervals) when estimating the future average value. The idea is to expand the joint probability distribution $p(\mathrm{y}, \overrightarrow{\mathrm{x}})$ in terms of local Gaussian models. Using the $x$ components at each site for definiteness, the value to be predicted $\mathrm{y}$ is taken as $x(n+2 \tau)$ (e.g., with embedding dimension $m=3$ and delay $\tau)$, given the vector of delayed components $\overrightarrow{\mathrm{x}}=\{x(n)$, $x(n+\tau), n\}$. The joint distribution is used to compute the average predicted values, $\langle y \mid \vec{x}\rangle$ and their variances, $\left\langle(y-\langle y \mid \vec{x}\rangle)^{2} \mid \vec{x}\right\rangle$. This provides a criterion for predictability since higher variance is associated with lower predictability. The use of uncertainty as measured by the variance is employed in Ref. [4] to obtain a definition of predictability associated with chaotic systems or stochastic processes, and such a method is used to distinguish between these two modes of evolution. Such a distinction has also been made in the context of time series analysis using the CWM approach [8].

\section{LOCAL DIMENSION IN SPATIOTEMPORAL CHAOTIC SYSTEMS}

\section{A. Coupled logistic maps}

We begin with the case of two-dimensional coupled map lattices consisting of logistic maps [9]:

$$
\begin{aligned}
x_{n+1}^{i, j}= & (1-\epsilon) f\left(x_{n}^{i, j}\right)+\frac{\epsilon}{4}\left[f\left(x_{n}^{i-1, j}\right)+f\left(x_{n}^{i+1, j}\right)\right. \\
& \left.+f\left(x_{n}^{i, j-1}\right)+f\left(x_{n}^{i, j+1}\right)\right],
\end{aligned}
$$

with

$$
f(x)=\mu x(1-x), \quad x \in(0,1), \quad \mu \in(0,4),
$$

where $i, j=1,2, \ldots, N$ and $\epsilon$ represents the coupling strength.

In this simulation, we use $N=50, \mu=4, \epsilon=0.4$, and periodic boundary conditions. The reference spatial variables $x^{i, j}, i, j=1,2 \ldots, N$, are obtained by evolving system (2) from random initial conditions. The number of iterations is chosen to be 5015 so that transients are removed. 


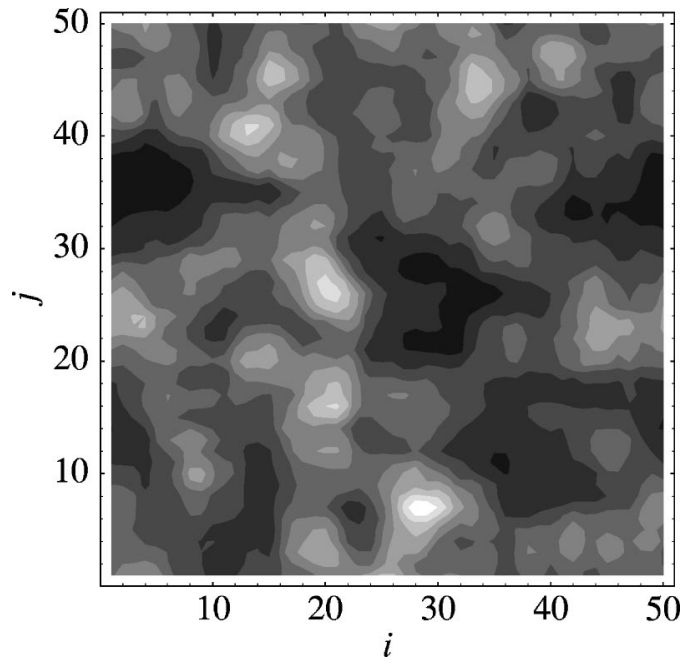

FIG. 2. Gray scale plot showing the regions of low (dark) and high (bright) local dimensions for the coupled logistic maps (2).

We generate additional spatial variables by adding small perturbations to the reference variables at time 5000 to measure the local instability of the coupled maps. Thus, at time 5015, we have spatial distributions corresponding to the reference variable and four distinct perturbations. The local dimension values at the spatial points are computed at this time value using the statistic defined in Eq. (1) as discussed in the preceding section. Figure 2 illustrates the results of the logistic maps (2) calculations where dark regions correspond to low dimensions and bright regions represent high dimensions. We found that the local dimension has a minimum value $\psi=1.0323$ at $i=2, j=37$ and a maximum value $\psi$ $=2.3636$ at $i=28, j=7$. We note that the maximum and minimum values at the spatial points $(i, j)$ are practically constant under evolution of system (2) a few steps forward or backward.

In order to establish a connection between dimension and predictability, we analyze the time series at the points $(2,37)$ and $(28,7)$ discussed above. We apply the simple prediction algorithm in the range from $n=5015$ to $n=5040$. Figure 3 shows the prediction errors, $\eta=\left|x_{\text {orig }}^{i, j}-x_{\text {pred }}^{i, j}\right|$, for $x^{2,37}$ and $x^{28,7}$ with $\delta=0.05$. It is evident that the prediction error is small at the lattice point $(2,37)$ where the local dimension is minimum, and large at the point $(28,7)$ where the local di-

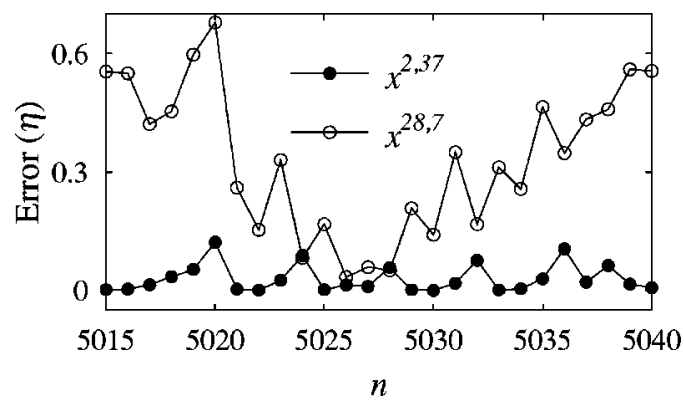

FIG. 3. Simple finite prediction error for the coupled logistic maps. The error remains minimum for $x^{2,37}$ (low BVD point) and high values of error obtained for $x^{28,7}$ (high BVD point).

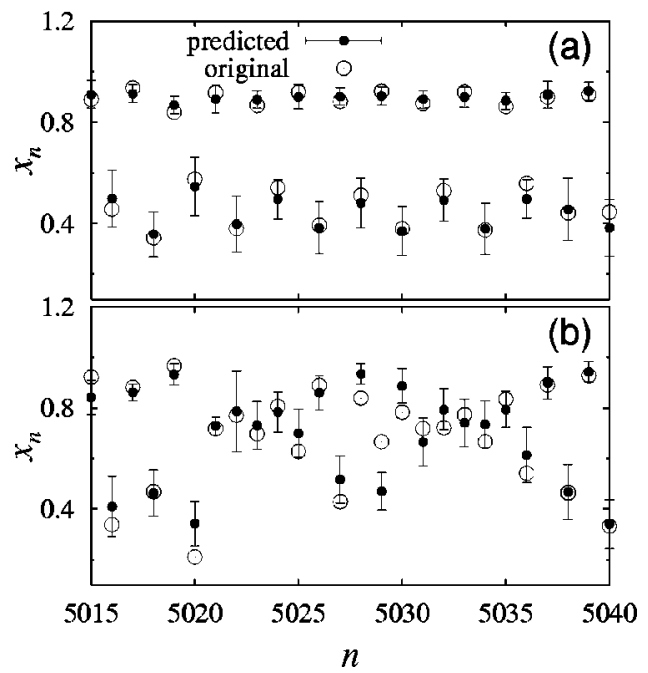

FIG. 4. Prediction of time series for the coupled logistic maps by using cluster weighted modeling. The original (circles) and predicted values (filled circles with error bars) of the time series at (a) low BVD location $(2,37)$ and (b) high BVD location $(28,7)$.

mension is maximum. In addition, similar results were obtained for points with other local dimension values. We found that the prediction error is consistently low in regions of low local dimension and high in the regions of relatively high local dimension.

The Bayesian approach, known as the cluster weighted modeling, will provide more insights into the predictability issue. Particularly important is the relationship between variance of predicted average value and the BV dimension. We consider the average prediction $\langle y \mid \vec{x}\rangle$ using the conditional distribution obtained from the joint distribution $p(\mathrm{y}, \overrightarrow{\mathrm{x}})$. As discussed in the preceding section, we take $\mathrm{y}$ to be the $x$ variable at time $n+2 \tau$ and $\overrightarrow{\mathrm{x}}=\left\{x_{n}, x_{n+\tau}, n\right\}$; here we take $\tau=1$. Figure 4 presents a series of predicted values, using always the two most recent original values, and the corresponding variances. In most analyzed cases, we found the following behavior. Lattice sites with high BV dimension result in predicted values with larger variances than those with low BV dimension, or either the prediction tends to fall outside the confidence interval defined by the variance of the future value. These facts are shown in Figs. 4(a) and 4(b) for the low and high BV dimensions, respectively 1.0323 and 2.3636. In this case, the simple prediction results are more compelling than the CWM since the difference between highest and lowest BV dimensions is not big enough. In the following section, we discuss another system where the maximum BV dimension is about four times larger than the minimum dimension over the spatial distribution. In this case, the conclusion that the uncertainty in prediction is related to dimension is even more forceful.

\section{B. Coupled Lorenz systems}

We next consider a two-dimensional array of diffusively coupled Lorenz oscillators represented by the following equations [10]: 


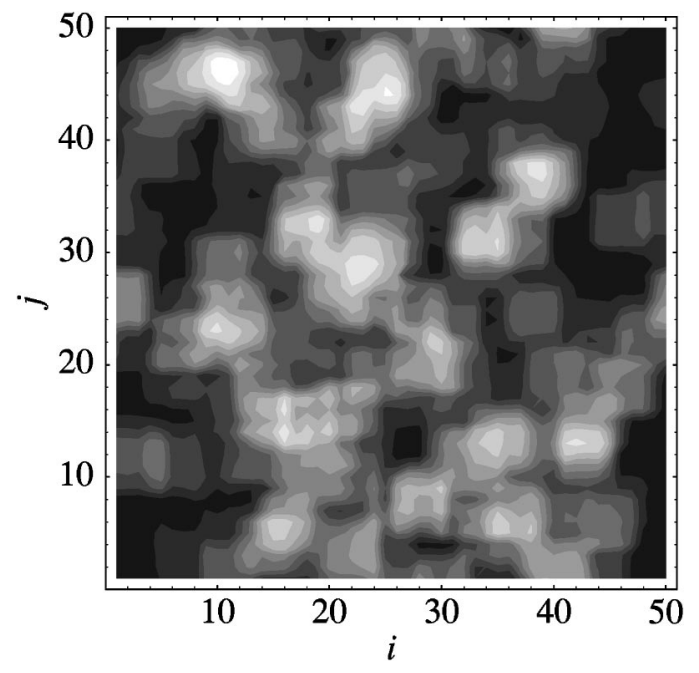

FIG. 5. Gray scale plot showing the regions of low (dark) and high (bright) local dimensions for the coupled Lorenz systems (4).

$$
\begin{gathered}
\dot{x}_{i, j}=\sigma\left(y_{i, j}-x_{i, j}\right)+c\left(y_{i-1, j}+y_{i+1, j}\right. \\
\left.+y_{i, j-1}+y_{i, j+1}-4 y_{i, j}\right), \\
\dot{y}_{i, j}=-x_{i, j} z_{i, j}+R x_{i, j}-y_{i, j}, \\
\dot{z}_{i, j}=x_{i, j} y_{i, j}-\beta z_{i, j},
\end{gathered}
$$

where $i, j=1,2, \ldots, N, \sigma=16, R=40, \beta=4$, and $c=1$. We use $50 \times 50(N=50)$ oscillators with periodic boundary conditions. Equations (4) are solved numerically using the fourth-order Runge-Kutta method with random initial conditions. We actually consider the $x$ and $y$ components representing a map of the above system by the Poincare section taken at $z=R-1$. The spatial distribution of the local dimensions is calculated in a similar fashion as in the case of coupled map lattices discussed earlier. A low value of $\psi=1.1447$ is found at lattice point $(i, j)=(3,50)$, while a high value $\psi=4.1226$ is computed at $(i, j)=(11,46)$. Figure 5 shows the regions of different local dimensions for the coupled Lorenz systems (4). The finite time prediction error in Fig. 6 uses $\delta \approx 0.8$ in the simple prediction algorithm. Here, the error is calculated as $\eta$

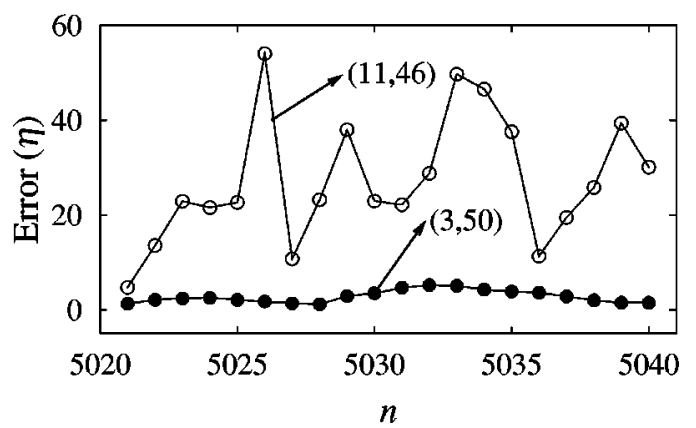

FIG. 6. Simple finite time prediction error for the coupled Lorenz systems. The absolute error remains minimum at $(3,50)$ for which the BV dimension is low, and high values of error obtained at $(11,46)$, the high BV dimension point.

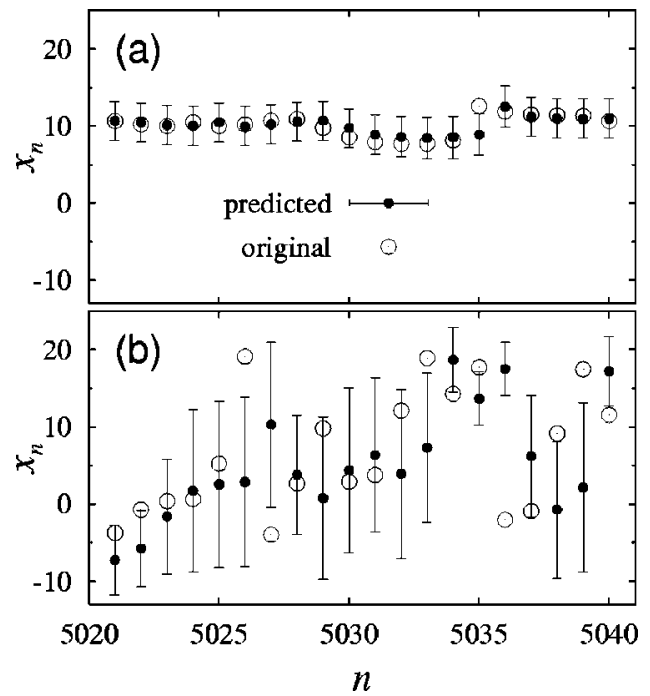

FIG. 7. Prediction of the time series using cluster weighted modeling at (a) $(3,50)$ and (b) $(11,46)$.

$=\sqrt{\left(x_{\text {orig }}-x_{\text {pred }}\right)^{2}+\left(y_{\text {orig }}-y_{\text {pred }}\right)^{2}}$. The results concerning the cluster weighted modeling are shown in Fig. 7. Here, high local dimension imply a consistent increase in the variances represented by larger error bars. In this case, there is a clear relationship between the predictability and the local BV dimension.

\section{SUMMARY AND CONCLUSIONS}

Predictability is related to the uncertainty in the outcome of future events during the evolution of the state of a system. We have interpreted the CWM as a tool to detect such an uncertainty and used it in spatially distributed systems. The simple prediction algorithm in conjunction with the CWM forms a powerful set of methods to relate predictability and dimension. Another tool based on the variance of future states of a system is also employed in Ref. [4] where a level of predictability is defined and applied to distinguish between deterministic and stochastic behaviors. Such a distinction requires a propagation time longer than the short time behavior used herein, and the cluster weighted modeling is more appropriate in the present context. Both methods, however, CWM or Ref. [4], are more than diagnostic tools and can be used to make real time predictions.

The short time evolution used here is not only a requirement for predicting chaotic systems but also guarantees the consistency of our conclusions. This comes about since the primary step is to identify spatial points of small BV dimension and then to make short time forecasts for the variables at these points. If the dimension changed substantially during the short time evolution then the relationship between dimension and prediction could not be maintained. In this work, predictions are made over intervals of 20 or 25 units of time (cycles) and under such circumstances the value of BV dimension is practically constant.

For deterministic evolution some systems are more predictable than others, and this can be measured by Lyapunov exponents. However, these exponents are well defined only 
asymptotically and are not unique for finite time calculations [11-14]. In such cases bred vectors are the proper tool to use, and in this paper we provided the connection between predictability and the value of the BV dimension.

The concept of bred vectors is intimately related to the analysis of geophysical flows $[1,15]$. Other forecasting techniques could be envisaged for real applications other than the CWM $[16,17]$. The main thesis of this work, however, will remain unaltered in such situations since the system is deterministic and the simple prediction algorithm or the CWM are essentially finite-dimensional maps. Other algorithms would at most imply some differences for instance in the error functions with no impact on the main conclusions. In a further investigation, we intend to apply the concept of BV dimension and the tools used herein to several configurations of fluids in the turbulent regime.

\section{ACKNOWLEDGMENT}

This work was supported by Fundação de Amparo à Pesquisa do Estado de São Paulo (FAPESP), Brazil.
[1] D.J. Patil, B.R. Hunt, E. Kalnay, J.A. Yorke, and E. Ott, Phys. Rev. Lett. 86, 5878 (2001).

[2] N. Gershenfeld, B. Schoner, and E. Metois, Nature (London) 397, 329 (1999).

[3] H. Kantz and T. Schreiber, Nonlinear Time Series Analysis (Cambridge University Press, Cambridge, 1997).

[4] L.W. Salvino, R. Cawley, C. Grebogi, and J.A. Yorke, Phys. Lett. A 209, 327 (1995).

[5] P. Grassberger and I. Procaccia, Physica D 9, 189 (1983).

[6] P. Grassberger and I. Procaccia, Phys. Rev. Lett. 50, 346 (1983).

[7] R. A. Johnson and D. W. Wichern, Applied Multivariate Statistical Analysis (Prentice Hall, New Jersey, 1998).

[8] F.F. Ferreira, G. Francisco, B.S. Machado, and P. Muruganandam, Physica A 321, 619 (2003).

[9] T. Bohr and O.B. Christensen, Phys. Rev. Lett. 63, 2161
(1989).

[10] L. Kocarev and U. Parlitz, Phys. Rev. Lett. 77, 2206 (1996).

[11] H.D.I. Abarbanel, R. Brown, and M.B. Kennel, Int. J. Mod. Phys. B 5, 1347 (1991).

[12] H.D.I. Abarbanel, R. Brown, and M.B. Kennel, J. Nonlinear Sci. 1, 175 (1991).

[13] J. Kurths and A. Brandenburg, Phys. Rev. A 44, R3427 (1991).

[14] S. Yoden and M. Nomura, J. Atmos. Sci. 50, 1531 (1993).

[15] E. Kalnay, Atmospheric Modeling, Data Assimilation, and Predictability (Cambridge University Press, Cambridge, 2002).

[16] T. Sauer, in Time Series Prediction: Forecasting the Future and Understanding the Past, edited by A. S. Weigend and N. A. Gershenfeld (Addision-Wesley, Reading, MA, 1994), pp. 175-193.

[17] E.A. Wan, in Time Series Prediction: Forecasting the Future and Understanding the Past (Ref. [16]), pp. 195-217. 